\title{
ANÁLISE DE RESULTADOS A PARTIR DE TESTES DE SENTENÇAS E QUESTIONÁRIO DE AUTO-AVALIAÇÃO
}

\author{
Results analysis based on tests of sentences \\ and a self-assessment questionnaire
}

\author{
Aline da Silva Lopes ${ }^{(1)}$, Nilvia Herondina Soares Aurélio ${ }^{(2)}$, \\ Sinéia Neujahr dos Santos ${ }^{(3)}$, Tiago Petry ${ }^{(4)}$, Maristela Júlio Costa ${ }^{(5)}$
}

\begin{abstract}
RESUMO
Objetivos: investigar a melhora no desempenho e na restrição de participação de usuários de próteses auditivas, após um período de três meses, e verificar se houve correlação entre os resultados obtidos nestes dois aspectos. Métodos: analisou-se 13 sujeitos, com perda auditiva do tipo neurossensorial de grau leve a moderadamente severo, com idades entre 28 e 60 anos. Realizou-se a pesquisa dos Limiares e Índices Percentuais de Reconhecimento de Sentenças no Silêncio e no Ruído (LRSS e LRSR, IPRSS e IPRSR), em campo livre, através do teste Listas de Sentenças em Português; e aplicou-se o questionário Hearing Handicap Inventory for Adults (HHIA). A primeira avaliação foi realizada antes da adaptação das próteses auditivas, sem o uso das mesmas, e a segunda, três meses após a adaptação, com o paciente fazendo uso das próteses auditivas. Resultados: a análise estatística evidenciou melhora significante, tanto em relação à restrição de participação (HHIA), quanto no desempenho das avaliações (LRSS, LRSR, IPRSS e IPRSR). Ao correlacionar a melhora na restrição de participação (HHIA), com a melhora do desempenho nos demais procedimentos, houve correlação significante apenas entre a melhora no HHIA e a melhora no LRSR. Conclusão: os usuários avaliados apresentaram sensação de restrição da participação diminuída, e melhora significante no desempenho em situações de reconhecimento de fala, tanto no silêncio quanto no ruído. Houve correlação entre a melhora nas respostas no HHIA e o LRSR, devido ao fato de que as maiores queixas em indivíduos com perda auditiva neurossensorial estarem relacionadas à presença de ruído competitivo.
\end{abstract}

DESCRITORES: Auxiliares de Audição; Testes de Discriminação da Fala; Questionários; Perda Auditiva

(1) Fonoaudióloga do Projeto Promoção da Saúde Auditiva da Universidade Federal de Santa Maria, UFSM, Santa Maria, RS; Mestranda em Distúrbios da Comunicação Humana pela Universidade Federal de Santa Maria.

(2) Fonoaudióloga do Projeto Promoção da Saúde Auditiva da Universidade Federal de Santa Maria, UFSM, Santa Maria, RS; Mestranda em Distúrbios da Comunicação Humana pela Universidade Federal de Santa Maria.

(3) Fonoaudióloga clínica da Sonora - Centro de Audiologia e Adaptação de Próteses Auditivas, Santa Maria, RS; Mestre em Distúrbios da Comunicação Humana pela Universidade Federal de Santa Maria.

(4) Fonoaudiólogo; Professor Assistente do Curso de Fonoaudiologia do Centro Universitário Feevale, FEEVALE, Novo Hamburgo, RS; Mestre em Distúrbios da Comunicação Humana pela Universidade Federal de Santa Maria.

(5) Fonoaudióloga; Professora Adjunta do Departamento de Fonoaudiologia da Universidade Federal de Santa Maria, UFSM, Santa Maria, RS; Doutora em Ciências dos Distúrbios da Comunicação Humana pela Universidade Federal de São Paulo

Conflito de interesses: inexistente

\section{INTRODUÇÃO}

Consta na literatura especializada que indivíduos com perda auditiva frequentemente se queixam de dificuldade de reconhecer a fala, principalmente na presença de ruído de fundo ${ }^{1}$.

As próteses auditivas são utilizadas como meio de amenizar a deficiência auditiva desses indivíduos, melhorando assim a sua qualidade de vida. Porém, apesar do avanço tecnológico dos sistemas de amplificação acústica modernos a satisfação dos usuários permanece um desafio e as taxas de abandono do uso de próteses continuam altas ${ }^{2}$.

A fim de avaliar a eficiência da reabilitação por meio das próteses auditivas, são usados métodos subjetivos como a audiometria tonal e vocal com a prótese para averiguar o ganho obtido ${ }^{3}$. No entanto, é de fundamental importância a medição 
da capacidade de reconhecimento da fala em situações mais próximas às reais, tais como testes audiológicos que empregam sentenças como estímulo ${ }^{4}$.

Porém, a satisfação do paciente não se relaciona apenas com o desempenho da prótese, mas também com o ponto de vista do mesmo, suas percepções e atitudes ${ }^{3}$.

Deve-se sempre considerar a existência de um período necessário de utilização da amplificação sonora para restabelecer as habilidades de fala e avaliar os benefícios obtidos com a amplificação, portanto a verificação da aclimatização sonora, considerando as habilidades de fala, uso, benefício e satisfação do usuário, são relevantes na prática clínica ${ }^{5}$.

Devido à importância da demonstração dos resultados na prática clínica, vários questionários de auto-avaliação tem sido desenvolvidos nos últimos anos, em um esforço para quantificar a percepção subjetiva de benefício trazido pelas próteses auditivas aos pacientes ${ }^{1}$. O uso de protocolos para avaliar o grau de satisfação do usuário é uma ferramenta simples e fácil de aplicar para registrar o desempenho da prótese, além de servir como um instrumento facilitador para ajudar em estratégia na orientação durante o período de aclimatização com a amplificação sonora ${ }^{6}$.

Existem duas filosofias diferentes referentes às técnicas de validação das próteses auditivas para documentar os resultados da adaptação: as que incidem sobre resultados subjetivos (utilizando questionários e entrevistas a fim de documentar as opiniões e atitudes do paciente) e as que incidem sobre os resultados objetivos (usando procedimentos empíricos para verificar as melhoras no desempenho) ${ }^{1}$.

Sendo assim, o objetivo desta pesquisa foi investigar a melhora no desempenho e na sensação de restrição de participação de adultos usuários de próteses auditivas, após um período de três meses, além de verificar a existência de correlação entre os resultados obtidos nos procedimentos objetivo e subjetivo.

\section{MÉTODOS}

Este estudo foi realizado no Laboratório de Próteses Auditivas do Serviço de Atendimento Fonoaudiológico (SAF) da Universidade Federal de Santa Maria (UFSM) a partir do projeto "Pesquisa em base de dados em saúde auditiva", registrado no Gabinete de projetos do Centro de Ciências da Saúde sob o no 019731.
Participaram do estudo somente indivíduos que concordaram com a realização dos procedimentos necessários para a execução da pesquisa e assinaram o Termo de Consentimento Livre e Esclarecido, após terem recebido maiores esclarecimentos sobre o objetivo e metodologia do estudo.

Para fazerem parte da pesquisa, os indivíduos deveriam ter idade entre 18 e 60 anos, diagnóstico audiológico de perda auditiva do tipo neurossensorial de grau leve a moderadamente severo, Limiar de Reconhecimento de Fala (LRF) de no máximo $65 \mathrm{~dB}$, nunca ter utilizado próteses auditivas, fazer parte do Programa de doação de próteses auditivas do Governo Federal, estar em processo de adaptação dos aparelhos auditivos e não ter qualquer fator que pudesse interferir no teste, como alterações neurológicas e/ou de fluência verbal.

Os sujeitos que participaram do estudo foram avaliados no período que compreendeu o mês de janeiro de 2008 a janeiro de 2009.

No total, fizeram parte do estudo 13 sujeitos, com idade média de 50 anos, com mínimo de 28 e máximo de 60 anos. Destes, 08 mulheres com média de idade de 46 anos, com mínimo de 28 e máximo de 60 anos e 05 homens com média de idade de 55 anos, com mínimo de 52 e máximo de 60 anos.

Os indivíduos foram submetidos à pesquisa dos Limiares de Reconhecimento de Sentenças no Silêncio e no Ruído (LRSS e LRSR) e dos Índices Percentuais de Reconhecimento de Sentenças no Silêncio e no Ruído (IPRSS e IPRSR), em campo livre. Além disso, foi aplicado o questionário de percepção de restrição de participação, Hearing Handicap Inventory for Adults (HHIA) (Figura 1). Os testes foram realizados em diferentes sessões de avaliação, consistindo em dois instantes para cada sujeito. A primeira avaliação foi realizada antes da adaptação das próteses auditivas, sem o uso das mesmas, e a segunda, três meses após a adaptação, com o paciente fazendo uso das próteses auditivas.

Obteve-se o LRSS, IPRSS, LRSR e o IPRSR através do teste Listas de Sentenças para o Português (LSP) ${ }^{4}$ (Figura 2), o qual é constituído por uma lista de 25 sentenças, outras sete listas com 10 sentenças e um ruído com espectro de fala. As sentenças e o ruído estão gravados em $C D$, em canais independentes.

Para obtenção das medidas foi utilizado um audiômetro digital de dois canais, marca Damplex, modelo DA65; e um sistema de amplificação para audiometria em campo livre, modelo TA 1010. As sentenças foram apresentadas utilizando-se um CD Player na marca Britânia, modelo B5279, na opção lineout acoplada ao audiômetro. 


\begin{tabular}{|c|c|c|c|}
\hline \multirow{2}{*}{\multicolumn{4}{|c|}{ S-1. A dificuldade em ouvir faz você usar o telefone menos vezes do que gostaria? }} \\
\hline & & & \\
\hline \multicolumn{4}{|l|}{$\begin{array}{l}\text { E-2. A dificuldade em ouvir faz você se sentir constrangido ou sem jeito quando é apresentado a pessoas } \\
\text { desconhecidas? }\end{array}$} \\
\hline \multicolumn{4}{|l|}{ S-3. A dificuldade em ouvir faz você evitar grupos de pessoas? } \\
\hline \multicolumn{4}{|l|}{ E-4. A dificuldade em ouvir faz você ficar irritado? } \\
\hline \multicolumn{4}{|l|}{$\begin{array}{l}\text { E-5. A dificuldade em ouvir faz você se sentir frustrado ou insatisfeito quando conversa com pessoas da sua } \\
\text { familia? }\end{array}$} \\
\hline \multicolumn{4}{|l|}{ S-6. A diminuição da audição causa dificuldades quando você vai a uma festa ou reunião social? } \\
\hline \multicolumn{4}{|l|}{ E-7. A dificuldade em ouvir faz você se sentir frustrado ao conversar com os colegas de trabalho? } \\
\hline \multicolumn{4}{|l|}{ S-8. Você sente dificuldade em ouvir quando vai ao cinema ou teatro? } \\
\hline \multicolumn{4}{|l|}{ E-9. Você se sente prejudicado ou diminuído devido a sua dificuldade em ouvir? } \\
\hline \multicolumn{4}{|l|}{ S-10. A diminuição da audição Ihe causa dificuldades quando visita amigos, parentes ou vizinhos? } \\
\hline \multicolumn{4}{|l|}{ S-11. A dificuldade em ouvir faz com que você tenha problemas para ouvir/entender os colegas de trabalho? } \\
\hline \multicolumn{4}{|l|}{ E-12. A dificuldade em ouvir faz você ficar nervoso? } \\
\hline \multicolumn{4}{|l|}{ S-13. A dificuldade em ouvir faz você visitar amigos, parentes ou vizinhos menos vezes do que gostaria? } \\
\hline \multicolumn{4}{|l|}{ E-14. A dificuldade em ouvir faz você ter discussōes ou brigas com a sua familia? } \\
\hline \multicolumn{4}{|l|}{ S-15. A diminuiçăo da audição Ihe causa dificuldades para assistir TV ou ouvir rádio? } \\
\hline \multicolumn{4}{|l|}{ S-16. A dificuldade em ouvir faz com que você saia para fazer compras menos vezes do que gostaria? } \\
\hline \multicolumn{4}{|l|}{ E-17. A dificuldade em ouvir deixa você de alguma maneira chateado ou aborrecido? } \\
\hline \multicolumn{4}{|l|}{ E-18. A dificuldade em ouvir faz você preferir ficar sozinho? } \\
\hline \multicolumn{4}{|l|}{ S-19. A dificuldade em ouvir faz você querer conversar menos com as pessoas da sua familia? } \\
\hline \multicolumn{4}{|l|}{ E-20. Você acha que a dificuldade em ouvir diminui ou limita de alguma forma sua vida pessoal ou social? } \\
\hline \multicolumn{4}{|l|}{$\begin{array}{l}\text { S-21. A diminuição da audição Ihe causa dificuldades quando você está em um restaurante com familiares ou } \\
\text { amigos? }\end{array}$} \\
\hline \multicolumn{4}{|l|}{ E-22. A dificuldade em ouvir faz você se sentir triste/deprimido? } \\
\hline \multicolumn{4}{|l|}{ S-23. A dificuldade em ouvir faz você assistir TV ou ouvir rádio menos vezes do que gostaria? } \\
\hline \multicolumn{4}{|l|}{$\begin{array}{l}\text { E-24. A dificuldade em ouvir faz você se sentir constrangido ou menos à vontade quando conversa com ami- } \\
\text { gos? }\end{array}$} \\
\hline E-25. A dificuldade em ouvir faz você se sentir isolado ou "deixado de lado" num grupo de pessoas? & & & \\
\hline
\end{tabular}

\section{Figura 1 - Hearing Handicap Inventory for Adults - Questionário para Avaliação do Handicap Auditivo em Adultos (Adaptação de ALMEIDA, 1998)}

Inicialmente foi realizado um treinamento para que os sujeitos se familiarizassem com o teste. As dez primeiras sentenças da lista $1 \mathrm{~A}$ foram aplicadas aos indivíduos antes da obtenção dos LRSS. Na obtenção dos LRSR foi utilizada a mesma tática, porém na presença de ruído competitivo.

A técnica para apresentação das sentenças foi baseada na estratégia denominada sequencial ou adaptativa ou ainda, ascendente-descendente ${ }^{7}$. Esta permite determinar o limiar de reconhecimento de fala, nível necessário para o indivíduo identificar de forma correta aproximadamente $50 \%$ dos estímulos apresentados.

O procedimento da pesquisa dos LRSS e LRSR consistiu na apresentação de um estímulo em uma determinada intensidade, obtida após o treino para a realização do teste. Se o indivíduo foi capaz de reconhecer corretamente o estímulo de fala apresentado, a intensidade do mesmo foi diminuída em intervalos preestabelecidos. Caso contrário, sua intensidade foi aumentada. Este procedimento foi repetido até o final da lista. O LRSR foi pesquisado com uma intensidade de ruído constante, a $65 \mathrm{~dB}$ (A) NPS.

Foram utilizados intervalos de $4 \mathrm{~dB}$ até a primeira mudança no tipo de resposta e, posteriormente, os intervalos de apresentação dos estímulos passaram a $2 \mathrm{~dB}$ entre si até o final da lista ${ }^{7}$.

$\mathrm{Na}$ pesquisa dos IPRSS e IPRSR, a intensidade das sentenças permaneceu sempre $65 \mathrm{~dB}(\mathrm{~A})$ NPS, intensidade esta escolhida em função de ser frequentemente utilizada pelas pessoas em situações habituais de conversação. No IPRSR, o ruído permaneceu constante a $65 \mathrm{~dB}$ (A) NPS, determinando uma relação $S / R$ fixa de zero.

As medidas em campo livre foram pesquisadas após a devida calibração do equipamento, por um profissional registrado no Inmetro SP, a fim de estabelecer os níveis de pressão sonora no qual o indivíduo testado perceberá a fala e ruído, tendo em vista as características do sinal de teste e as condições acústicas do ambiente. Para isso, foi utilizado 


\section{LISTA 1A}

1. Não posso perder o ônibus.

2. Vamos tomar um cafezinho.

3. Preciso ir ao médico.

4. A porta da frente está aberta.

5. A comida tinha muito sal.

6. Cheguei atrasado para a reunião.

7. Vamos conversar lá na sala.

8. Depois liga pra mim.

9. Esqueci de pagar a conta.

10. Os preços subiram ontem.

11. O jantar está na mesa.

12. As crianças estão brincando.

13. Choveu muito neste fim-de-semana.

14. Estou morrendo de saudade.

15. Olhe bem ao atravessar a rua.

16. Preciso pensar com calma.

17. Guardei o livro na primeira gaveta.

18. Hoje é meu dia de sorte.

19. O sol está muito quente.

20. Sua mãe acabou de sair de carro.

\section{LISTA 3B}

1. Ela acabou de bater o carro.

2. É perigoso andar nessa rua.

3. Não posso dizer nada.

4. A chuva foi muito forte.

5. Os preços subiram na segunda.

6. Esqueci de levar a bolsa.

7. Os pães estavam quentes.

8. Elas já alugaram uma casa na praia.

9. Meu irmão viajou de manhã.

10. Não encontrei meu filho.

\section{LISTA 4B}

1. Sua mãe pôs o carro na garagem.

2. O aluno quer assistir ao filme.

3. Ainda não pensei no que fazer.

4. Essa estrada é perigosa.

5. Não paguei a conta do bar.

6. Meu filho está ouvindo música.

7. A chuva inundou a rua.

8. Amanhã não posso almoçar.

9. Ela viaja em dezembro.

10. Você teve muita sorte.

\section{LISTA 5B}

\section{LISTA 1B}

1. O avião já está atrasado.

2. O preço da roupa não subiu.

3. O jantar da sua mãe estava bom.

4. Esqueci de ir ao banco.

5. Ganhei um carro azul lindo.

6. Ela não está com muita pressa.

7. Avisei seu filho agora.

8. Tem que esperar na fila.

9. Elas foram almoçar mais tarde.

10. Não pude chegar na hora.

\section{LISTA 2B}

1. Acabei de passar um cafezinho.

2. A bolsa está dentro do carro.

3. Hoje não é meu dia de folga.

4. Encontrei seu irmão na rua.

5. Elas viajaram de avião.

6. Seu trabalho estará pronto amanhã.

7. Ainda não está na hora.

8. Parece que agora vai chover.

9. Esqueci de comprar os pães.

10. Ouvi uma música linda.
1. Depois, a gente conversa.

2. Ela acabou de servir o almoço.

3. Esta carta chegou ontem.

4. Preciso terminar o meu trabalho.

5. Não posso esquecer da mala.

6. A rua estava muito escura.

7. A data do exame foi adiada.

8. Elas alugaram um carro no verão.

9. Minha viagem foi ótima.

10. Eles foram comprar pães.

\section{LISTA 6B}

1. Vou viajar as nove da manhã.

2. Meu irmão bateu o carro ontem.

3. Prometi a ele não contar o segredo.

4. Cheguei atrasada na aula.

5. Esta rua é perigosa.

6. Esqueci da bolsa na sua mesa.

7. Ela comprou os últimos pães.

8. A casa de campo já foi alugada.

9. Os preços não devem subir.

10. Não falei com sua filha.

Figura 2 - Listas de Sentenças em português utilizadas 
Microfone B\&K 4144 n/s, Medidor de Nível de Pressão Sonora B\&K 2260 n/s e Calibrador de Nível de Pressão Sonora B\&K n/s. O medidor foi posicionado em um ponto médio entre as duas orelhas, a uma distância de um metro do alto-falante.

O VU-meter do audiômetro foi colocado na posição 0 antes de cada avaliação e cada canal do CD foi calibrado separadamente, sendo que um tom puro de $1000 \mathrm{~Hz}$ foi utilizado para calibrar o canal das sentenças. $O$ uso do tom puro é necessário, pois a fala é um som complexo, que apresenta uma variação de $30 \mathrm{~dB}$ entre o som mais intenso e o menos intenso, oscilando $12 \mathrm{~dB}$ acima e 18 $\mathrm{dB}$ abaixo da média ${ }^{8}$. Portanto, o uso de um som contínuo de referência mantém sempre as mesmas condições de apresentação. Já o canal do ruído foi calibrado usando o próprio ruído, gravado no outro canal.

Foi usado um medidor digital do nível de pressão sonora (NPS), marca Radio Schack periodicamente para monitorar os níveis de apresentação dos diferentes estímulos, determinando e garantindo sempre as mesmas condições acústicas no campo livre para todos os sujeitos avaliados.

O questionário de percepção da restrição de participação, HHIA, foi desenvolvido em $1995^{9} \mathrm{e}$ adaptado ao português em $1998{ }^{10}$ para avaliar o impacto da perda auditiva nos aspectos sociais e emocionais do indivíduo adulto. Através das respostas às questões relacionadas à sua audição, os pacientes reconhecem se a situação representa um problema.

A pontuação obtida no questionário é analisada da seguinte maneira: a resposta negativa é equivalente a zero, "às vezes" equivale a dois, e a resposta positiva vale quatro. As respostas obtidas foram analisadas e as pontuações encontradas, padronizadas, ou seja, transformadas em índices percentuais, indicando seu desempenho. $O$ valor da pontuação poderia variar de 0 a 100, sendo que quanto maior o valor obtido, maior a autopercepção da restrição de participação. Resultados inferiores a 16 pontos representam não haver percepção do handicap, de 18 a 42 pontos, indicam uma percepção leve a moderada e acima de 42 pontos, uma percepção severa ou significativa. Pelo fato de o questionário ter sido aplicado na $1^{\underline{a}}$ e $2^{\underline{a}}$ sessão, ajuda-nos na avaliação dos benefícios do uso da amplificação, permitindo a verificação da mudança na autopercepção da restrição de participação.

Este estudo foi aprovado pela Comissão de Ética em Pesquisa (CEP) da UFSM em 05/12/2006, protocolo $\mathrm{n}^{\circ}$ 23081.0.016862/2006-09.

As variáveis consideradas neste estudo foram LRSS $\left(1^{\circ}\right.$ e $\left.2^{\circ}\right)$, LRSR $\left(1^{\circ}\right.$ e $2^{\circ}-$ expressos sob a forma de relação $S / R)$, IPRSS $\left(1^{\circ}\right.$ e $\left.2^{\circ}\right)$, IPRSR $\left(1^{\circ}\right.$ e $\left.2^{\circ}\right)$ e respostas ao questionário $\mathrm{HHIA}\left(1^{\circ}\right.$ e $\left.2^{\circ}\right)$. Após verificar a distribuição dos dados, constatou-se que apenas o $2^{\circ}$ IPRSS e o $2^{\circ}$ HHIA apresentaram distribuição não-normal, sendo que para estas variáveis foi utilizado o teste estatístico não-paramétrico de Wilcoxon. Para todas as demais variáveis, as quais apresentaram distribuição normal, foi aplicado o teste para a diferença entre duas amostras dependentes, o teste t pareado, que verifica se as diferenças encontradas entre os instantes de avaliação, tiveram significância ou não. Ainda, foi efetuada uma análise descritiva dos dados, mostrando os resultados entre as sessões de avaliação, além da análise da correlação entre as melhoras de variáveis diferentes, na qual foi utilizado o Coeficiente de Correlação de Spearman.

As variáveis foram comparadas duas a duas, em diferentes sessões de avaliação: $1^{a}$ sessão - antes da adaptação dos aparelhos auditivos, sem o uso dos aparelhos auditivos e; $2^{2}$ sessão - três meses após a adaptação dos aparelhos auditivos, com o paciente fazendo uso dos mesmos. Foi considerado resultado significante $p<0,05(5 \%)$.

\section{RESULTADOS}

Na Tabela 1 estão apresentados a média, desvio padrão, valor máximo, mínimo e os resultados do teste t pareado ou Wilcoxon entre as sessões de avaliação, para as variáveis LRSS, Relação S/R, IPRSS, IPRSR e HHIA.

$\mathrm{Na}$ Tabela 2 estão o percentual de indivíduos que melhoraram e os valores de melhora entre as avaliações.

Na Tabela 3 estão apresentadas as correlações entre a melhora na sensação de restrição de participação, através do HHIA, com a melhora do desempenho dos indivíduos nos demais procedimentos (LRSS, relação S/R, IPRSS e IPRSR), através do Coeficiente de Correlação de Spearman.

Vale ressaltar que no IPRSS, nove indivíduos apresentaram $100 \%$ de respostas corretas na primeira avaliação, devido ao fato de o procedimento ter sido realizado na intensidade de $65 \mathrm{~dB}$ (A) NPS, e assim, os quatro restantes puderam ser analisados com relação à melhora e apresentaram índices de $100 \%$ na segunda avaliação. Dessa forma, na Tabela 3, a variável melhora no IPRSS não foi analisada estatisticamente, pelo pequeno número de indivíduos. É importante observar que para fazer a correta apreciação dos resultados, quanto menores os valores das variáveis, melhor o desempenho do indivíduo (com exceção dos itens IPRSS e IPRSR, nos quais os maiores valores demonstram o melhor desempenho do indivíduo). 
Tabela 1 - Média, desvio padrão, valores mínimos, máximos e resultado do teste t pareado ou Wilcoxon, entre as sessões de avaliação, para o LRSS, Relação S/R, IPRSS, IPRSR e HHIA

\begin{tabular}{|c|c|c|c|c|c|c|}
\hline Variáveis & Sessão & Média & DP & Mín & Máx & Diferença \\
\hline \multirow[t]{2}{*}{ LRSS } & $1^{\mathrm{a}}$ & 55,54 & 7,63 & 45,14 & 66,78 & \multirow{2}{*}{$0,0006^{\star *}$} \\
\hline & $2^{a}$ & 45,00 & 8,08 & 32,20 & 57,86 & \\
\hline \multirow[t]{2}{*}{ Rel S/R } & $1^{\mathrm{a}}$ & 2,29 & 3,86 & $-5,78$ & 7,33 & \multirow{2}{*}{$0,0001^{* *}$} \\
\hline & $2^{\mathrm{a}}$ & $-1,92$ & 3,14 & $-8,29$ & 5,56 & \\
\hline \multirow[t]{2}{*}{ IPRSS } & $1^{\mathrm{a}}$ & 86,92 & 23,23 & 40,00 & 100,00 & \multirow{2}{*}{$0,0464^{*}$} \\
\hline & $2^{\underline{a}}$ & 100,00 & 0 & 100,00 & 100,00 & \\
\hline \multirow[t]{2}{*}{ IPRSR } & $1^{\mathrm{a}}$ & 49,23 & 28,12 & 0,00 & 100,00 & \multirow{2}{*}{$0,0003^{* *}$} \\
\hline & $2^{\underline{a}}$ & 77,69 & 19,64 & 40,00 & 100,00 & \\
\hline \multirow[t]{2}{*}{ HHIA } & $1^{\mathrm{a}}$ & 60,46 & 21,51 & 16,00 & 94,00 & \multirow{2}{*}{$0,0016^{\star}$} \\
\hline & $2^{\underline{a}}$ & 26,46 & 21,84 & 4,00 & 86,00 & \\
\hline
\end{tabular}

${ }^{*}$ Resultados do teste estatístico não-paramétrico de Wilcoxon

${ }^{* *}$ Resultados do teste estatístico paramétrico t pareado

***Todas as análises apresentaram diferença estatisticamente significante $(p<0,05)$

Tabela 2 - Percentual de sujeitos que melhoraram e os valores de melhora entre as sessões de avaliação dos LRSS, relações S/R, IPRSS, IPRSR e HHIA

\begin{tabular}{|c|c|c|c|}
\hline Variáveis & $\mathbf{N}$ & Melhoraram & Valores médios de melhora \\
\hline $1^{\circ}$ LRSS $\times 2^{\circ}$ LRSS & 13 & $100 \%$ & $10,54 \mathrm{~dB}$ \\
\hline $1^{\circ} \mathrm{Rel} S / \mathrm{R} \times 2^{\circ}{ }^{\circ} \mathrm{Rel} S / \mathrm{R}$ & 12 & $92,31 \%$ & $4,21 \mathrm{~dB}$ \\
\hline $1^{\circ}$ IPRSS $\times 2^{\circ}$ IPRSS* & 04 & $30,77 \%$ & $42,50 \%$ \\
\hline 1 ㅇ IPRSR X 2 IPRSR & 12 & $92,31 \%$ & $28,46 \%$ \\
\hline $1^{\circ} \mathrm{HHIA} \times 2^{\circ} \mathrm{HHIA}$ & 12 & $92,31 \%$ & 34 pontos \\
\hline
\end{tabular}

* No IPRSS, nove indivíduos apresentaram $100 \%$ de respostas corretas já na primeira avaliação.

Tabela 3 - Correlações entre as melhoras no HHIA e os demais procedimentos (LRSS, relação S/R e IPRSR)

\begin{tabular}{cccc}
\hline Variável & LRSS & Rel S/R & IPRSR \\
\hline HHIA & $\mathrm{p}=0,5902$ & $\mathrm{p}=-0,0421^{\star}$ & $\mathrm{p}=-0,8826$ \\
\hline
\end{tabular}

*Apresentou correlação positiva estatisticamente significante $(p>0,05)$

\section{DISCUSSÃO}

Ao analisar estatisticamente os resultados das avaliações objetivas e do questionário de auto-avaliação encontrados na $1^{a}$ sessão (sem o uso das próteses auditivas) e na $2^{\text {a }}$ sessão (três meses após a adaptação das próteses auditivas, fazendo uso dos mesmos), foi verificada diferença significante entre as medidas obtidas nas duas sessões, observando-se melhorias significativas em termos de desempenho em todos os aspectos pesquisados.

Considerando o percentual de indivíduos em que foi possível constatar modificações entre as sessões de avaliação, observou-se que, tanto nas avaliações objetivas quanto na avaliação subjetiva, todos ou grande parte dos indivíduos avaliados apresentaram melhora nos aspectos analisados. 
Estes dados evidenciam uma melhora progressiva nas habilidades auditivas após um período de uso das próteses auditivas. Percebe-se então, a presença de uma recuperação auditiva nestes novos usuários de próteses auditivas, onde o quadro da privação auditiva foi interrompido, com uma melhora significativa nas habilidades de fala após três meses de uso da amplificação.

Em estudos anteriores ${ }^{10,11}$ foi relatado o fenômeno da aclimatização como o período que sucede a adaptação dos amplificadores sonoros, quando ocorre uma melhora progressiva das habilidades auditivas e reconhecimento de fala, decorrente das novas pistas de fala disponíveis ao usuário da amplificação, após um período de seis a 12 semanas de uso da amplificação.

Em outro estudo ${ }^{5}$, a avaliação objetiva, por meio de tarefas de reconhecimento de fala, indicou melhores resultados nos meses subseqüentes à adaptação das próteses auditivas evidenciando melhora progressiva das habilidades de fala a partir do primeiro mês de adaptação

Os resultados dos estudos citados acima corroboram com os achados da presente pesquisa, pois, em ambos, os sujeitos apresentaram um aprimoramento significativo nas habilidades de reconhecimento de fala.

Vale ressaltar que na pesquisa do IPRSS, o percentual de indivíduos que obtiveram melhora entre a $1^{a}$ e $2^{a}$ sessão foi de $30,77 \%$. Porém, este baixo percentual deve-se ao fato já elucidado no capítulo de resultados, em que os demais indivíduos já apresentaram um índice de 100\% de acertos, na $1^{\text {a }}$ sessão, pois o procedimento foi realizado a uma intensidade de $65 \mathrm{~dB}$ (A) NPS. Isso nos sugere que a intensidade para a obtenção do índice percentual deve ser baseada no resultado encontrado na pesquisa do limiar, a fim de as medidas de índice realizadas com e sem prótese nos mostrem resultados mais fidedignos.

Analisando os valores de melhora entre as avaliações, encontrou-se, no silêncio, para o LRSS, um valor médio de melhora de $10,54 \mathrm{~dB}$ entre a $1^{\mathrm{a}}$ e a $2^{\mathrm{a}}$ sessão de avaliação.

$\mathrm{Na}$ pesquisa do IPRSS, a média de melhora encontrada foi de $42,50 \%$ entre a $1^{\text {a }}$ e a $2^{2}$ sessão. Estes dados mostram uma clara mudança no reconhecimento de fala pelo uso das próteses auditivas na condição silêncio, uma vez que este resultado indica que em uma situação de conversação realizada no silêncio, o indivíduo pode ter uma melhora próxima a $50 \%$ de reconhecimento das sentenças, o que resulta em uma possibilidade de melhora substancial na compreensão do discurso.

Para a situação com ruído, a média de melhora foi de $4,21 \mathrm{~dB}$ entre a $1^{\underline{a}}$ e a $2^{\underline{a}}$ relação $S / R$.
Considerando que na literatura consultada ${ }^{12}$, realizada utilizando o mesmo material da presente pesquisa, foi referido que a mudança de apenas 1 $\mathrm{dB}$ na relação $\mathrm{S} / \mathrm{R}$, pode ocasionar uma mudança de aproximadamente $11,20 \%$ no reconhecimento de fala em indivíduos com perda auditiva. Com base nisso, é possível fazer uma projeção que, se os indivíduos foram capazes de reconhecer, na segunda sessão de avaliação, em torno de $50 \%$ dos estímulos apresentados em uma condição mais desfavorável, pois a fala foi apresentada em torno de $4 \mathrm{~dB}$ abaixo do ruído e multiplicarmos isso pela porcentagem de melhora citada pela autora acima, pode-se sugerir que os indivíduos tem uma possibilidade de melhorar a performance em torno de $47 \%$, com esta mudança na relação $S / R$, o que é um valor bastante expressivo, pois possibilitaria que eles tivessem respostas próximas a $100 \%$.

Em relação aos índices na presença de ruído competitivo, obteve-se uma média de melhora de $28,46 \%$ entre o $1^{\circ}$ e o $2^{\circ}$ IPRSR. Esses resultados são de extrema importância quando o transpomos para uma situação conversacional, onde o ruído competitivo está presente e podemos observar que há uma melhora de quase $30 \%$ na inteligibilidade de fala, mesmo quando as condições de ruído se mantiveram, mostrando que foram as habilidades auditivas que proporcionaram esta melhora.

$\mathrm{Na}$ literatura consultada ${ }^{13}$, enfatiza-se que - sucesso na adaptação de próteses auditivas envolve múltiplos aspectos, portanto não podemos restringir os benefícios da aclimatização apenas à melhora no reconhecimento de fala, mas também aos aspectos que envolvem toda a comunicação do usuário e propicie a ele um nível de satisfação quanto a sua nova condição de ouvinte.

Dessa forma, considerando então os resultados na avaliação subjetiva (HHIA), verificou-se que houve mudança nas respostas entre a primeira e segunda sessão de avaliação, uma vez que a autopercepção da restrição de participação passou de "severa ou significativa" para "leve ou moderada". Assim, pôde-se observar que grande parte das desvantagens emocionais e sociais geradas pela perda auditiva nos indivíduos avaliados foi diminuída com o uso da amplificação sonora.

Outros estudos 13,14 também encontraram evidências da aclimatização de forma significativa, principalmente por meio de medidas subjetivas (questionário de auto-avaliação). Em uma revisão da literatura realizada em 2005, o autor ${ }^{15}$ relatou que os benefícios observados pareceram estar relacionados à redução da percepção da desvantagem, melhora na qualidade de vida, melhor utilização das próteses auditivas e das estratégias de 
comunicação e alguma melhora no ajuste pessoal, indo ao encontro dos achados do presente estudo.

Alguns autores ${ }^{16}$ ressaltaram a importância da reavaliação após um período um pouco mais longo de experiência com as próteses auditivas, pois parece ocorrer uma diminuição do deslumbramento inicial e, neste momento, os pacientes conseguem se expressar de uma maneira mais precisa e consciente.

Nesta pesquisa, a reavaliação foi realizada após três meses de uso das próteses auditivas, portanto o deslumbramento inicial citado pelos autores acima pode ter sido atenuado.

Finalmente, ao comparar as melhoras obtidas através das diferentes estratégias de avaliação (LRSS, relação S/R e IPRSR) e os resultados no questionário HHIA, pôde ser observado que houve correlação significante apenas entre a melhora no $H H I A$ e a melhora na relação $S / R$, indicando que à medida que o desempenho no LRSR melhora, a pontuação no HHIA também melhora. Atribui-se esse achado, ao fato de que as maiores queixas de restrição de participação em indivíduos com perda auditiva neurossensorial estão relacionadas à presença de ruído competitivo.

O fato de não ter havido correlação significante entre todas as medidas objetivas e o questionário de auto-avaliação sugere que, a pontuação do HHIA, por ser uma avaliação subjetiva, está diretamente relacionada com as expectativas e motivação individuais. Esse resultado significa que muitas vezes o indivíduo pode apresentar resultados bastante satisfatórios do ponto de vista audiológico, mas para ele aquele benefício não é o suficiente e vice-versa.

Por exemplo, um indivíduo que tem muita expectativa, ou que supervaloriza qualquer dificuldade de comunicação encontrada, vai ter também mais dificuldade de se sentir satisfeito com o resultado obtido. Por outro lado, os indivíduos que sentem muita vontade de fazer uso das próteses e ainda sentem-se gratos por estarem recebendo este benefício gratuitamente, podem não se sentir à vontade para externar que não estão satisfeitos com os benefícios que as próteses estão oferecendo.

Pesquisas relacionadas à satisfação de usuários de próteses auditivas ${ }^{17,18}$ apontam que 0 sucesso da amplificação depende de vários fatores, tais como idade do usuário, o grau da perda auditiva, a compreensão da fala, a tolerância para sons intensos, as expectativas e a motivação do indivíduo, além da aceitação da perda auditiva.

Quantificações objetivas e subjetivas de melhorias no desempenho do reconhecimento de fala devem ser documentadas no processo de avaliação das próteses auditivas para verificar as melhorias ocorridas. Em última análise, esses resultados podem ajudar a proporcionar uma maior padronização para os procedimentos utilizados no processo de avaliação das próteses auditivas em diversas clinicas ${ }^{1}$.

Por este motivo, é fundamental a realização de procedimentos objetivos e subjetivos para reforçar a bateria de testes para avaliação das próteses auditivas. Dessa forma é possível verificar, nos casos em que um dos dois aspectos não está fornecendo dados satisfatórios, qual seria a estratégia a ser tomada a fim de minimizar a sensação de restrição de participação do indivíduo.

Além disso, torna-se ainda mais evidente que é importante ficar atento às expectativas do paciente antes de iniciar o processo de seleção e adaptação de próteses auditivas, deixando claro o que é possível e o que não vai ser possível restabelecer com o uso das mesmas, orientando e acompanhando o processo de adaptação e, também, realizando a revisão das regulagens, ou sugerindo reabilitação para os casos em que o resultados não são satisfatórios.

\section{CONCLUSÃO}

Após o período de aclimatização (três meses), concluiu-se que os indivíduos pesquisados apresentaram sensação de restrição da participação diminuída, e o desempenho em situações de reconhecimento de fala, tanto no silêncio quanto no ruído, apresentou melhora significante.

Além disso, houve correlação entre a melhora nas respostas no HHIA e o desempenho no ruído, corroborando o fato de que as maiores queixas de restrição de participação em indivíduos com perda auditiva neurossensorial são com relação à presença de ruído competitivo. 


\section{ABSTRACT}

Purpose: to investigate the improvement in the performance and in the participation restriction of hearing aids users after a three-month period, as well as to find out a possible correlation between the results obtained in these two aspects. Methods: 13 subjects having mild to moderate-severe sensorineural hearing loss, aged between 28 and 60 year old, were analyzed. The research of Sentence Recognition Threshold and Percent Indexes in Quiet and in Noise (SRTQ and SRTN, PISRQ and PISRN) was carried out in sound field through the Portuguese Sentences Lists test. The Hearing Handicap Inventory for Adults (HHIA) questionnaire was also applied. The first assessment was performed before the hearing aids fitting, without patients using them; the second, three months later, with the patients using the hearing aids. Results: the statistical analysis showed a significant improvement not only in relation to participation restriction (HHIA) but also in the performance of assessments (SRTQ, SRTN, PISRQ PISRN). When correlating the improvement in participation restriction ( $\mathrm{HHIA}$ ) with the improvement in the other procedures, there was a significant correlation only between the improvement in HHIA and the improvement in SRTN. Conclusion: the assessed users showed a feeling of decreased participation restriction, and a performance improvement in situations of speech recognition in quiet as well as in noise. There was a correlation between an improvement in the answers to HHIA and SRTN due to the fact that most complaints in people with sensorineural hearing loss are related to the presence of competitive noise.

KEYWORDS: Hearing Aids; Speech Discrimination Tests; Questionnaires; Hearing Loss

\section{REFERÊNCIAS}

1. Mendel LL Objective and subjective hearing aid assessment outcomes. Am J Audiol. 2007; 16(2):118-29.

2. Soares DO, Tavares RA, Ferreira RT, Guglielmino G, Dinato $C$, Franchi VM. Satisfação dos usuários de prótese auditiva em seu dia-a-dia. ACTA ORL. 2007; 25(4):290-2.

3. Veiga LR, Merlo ARC, Mengue SS. Satisfação com a prótese auditiva na vida diária em usuários do sistema de saúde do Exército. Rev Bras Otorrinolaringol. 2005; 71(1):67-73.

4. Costa MJ. Listas de sentenças em português: apresentação e estratégias de aplicação na audiologia. Santa Maria: Pallotti; 1998.

5. Prates LPCS, lório MCM. Aclimatização: estudo do reconhecimento de fala em usuários de próteses auditivas. Pró-Fono. 2006; 18(3):259-66.

6. Teixeira CF, Augusto LGS, Caldas Neto SS. Prótese auditiva: satisfação do usuário com sua prótese e com seu meio ambiente. Rev CEFAC. 2008; 10(2):245-53.

7. Levitt $H$, Rabiner LR. Use of a sequential strategy in intelligibility testing. J Acoust Soc Am. 1967; 42(3):609-12.

8. Boothroyd A. Speech perception, sensorioneural hearing loss and hearing aid. In: Studevaker G, Hochberg I. Acoustical factors affeting hearing aid performance. Boston: Allyn \& Bacon; 1993. p. 277-99.
9. Cox RM, Alexander GC. The abbreviated profile of hearing aid benefit. Ear Hear. 1995; 16(2):176-83. 10. Almeida K. Avaliação dos resultados da intervenção. In: Almeida K, lório MCM. Próteses auditivas: fundamentos teóricos \& aplicações clínicas. 2. ed. São Paulo: Lovise; 2003. p.335-53.

11. Munro KJ, Lutman ME. The effect of speech presentation level on measurement of auditory acclimatization to amplified speech. J Accoust Soc Am. 2003; 114(1):484-95.

12. Henriques MO. Limiares e índices percentuais de reconhecimento de sentenças no ruído, em campo livre, para indivíduos adultos [dissertação]. Santa Maria (RS): Universidade Federal de Santa Maria; 2006.

13. Humes LE, Wilson DL, Barlow NH, Garner C. Changes in hearing-aid benefit following 1 or 2 years of hearing-aids use by older adults. J Speech Lang Hear Res. 2002; 45(4):772-82.

14. Bucuvic EC, lório MCM. Benefício e dificuldades auditivas: um estudo em novos usuários de próteses auditivas após dois e seis meses de uso. Fono Atual. 2004; 29(7):19-29.

15. Hawkins EA. Effectiveness of counselingbased adult group aural rehabilitation programs: a systematic review of the evidence. J Am Acad Audiol. 2005; 16(7):485-93.

16. Amorim RMC, Almeida K. Estudo do benefício e da aclimatização em novos usuários de próteses auditivas. Pró-Fono. 2007; 19(1):39-48. 
74 Lopes AS, Aurélio NHS, Santos SN, Petry T, Costa MJ

17. Batista ACM, Sampaio FM. Nível de satisfação de idoso usuários de próteses auditivas doadas pela APAC-NAMI-UNIFOR. RBPS: Rev Bras Prom Saúde. 2005; 18(7):7-10.
18. Costa MHP, Sampaio ALL, Oliveira CACP. Avaliação do benefício da prótese auditiva digital e da percepção da desvantagem auditiva ou "handicap" em idosos não institucionalizados. Arq Int de Otorrinolaringol. 2007; 11(2)159-68.

RECEBIDO EM: 20/08/2009

ACEITO EM: 07/07/2010

Endereço para correspondência:

Aline da Silva Lopes

Rua Mal. Floriano Peixoto, 878 ap. 05

Santa Maria - RS

CEP: 97010-001

E-mail: lopezas@gmail.com

Rev. CEFAC. 2011 Jan-Fev; 13(1):65-74 